\title{
Effects of prior activity on the conduction in single motor units in man
}

\author{
JÖRGEN BORG \\ From the Department of Neurology, Karolinska Hospital, Stockholm, Sweden
}

SUMMARY The slowing of the conduction of an impulse travelling in the relative refractory period of a prior impulse was studied in single short toe extensor motor units in man. Paired electrical stimuli were delivered to the peroneal nerve and single motor unit recordings were achieved by special electromyographic techniques. The delay of the second impulse, propagated during the relative refractory period of the prior impulse, limited the unit discharge frequency. The shortest motor unit response interval ranged from 3 to $5 \mathrm{~ms}$ and was inversely related to the axonal conduction velocity. The delay of the second impulse in the proximal nerve segment was most pronounced close to the stimulus point but continued during propagation for several milliseconds. Further delay occurred distally. The slowing of the conduction of the second impulse after delivering paired electrical nerve stimuli offers a new parameter in single motor unit studies and also makes it possible to measure the axonal refractory period after electrical nerve excitation without using blocking nerve impulses.

It is known from animal experiments that the conduction velocity of a nerve impulse travelling in the relative refractory period of a prior impulse is lower than when travelling in the nerve fibre at rest. ${ }^{1}$ In a human peripheral nerve the slowing of the second impulse volley has been used as an indicator of the relative refractory period of the nerve trunk,,$^{23}$ but no single nerve fibre study has been made in man.

In previous studies in man, the refractory period of single alpha motor axons was measured using highly selective electromyographic recordings (cf Methods). The excitability changes following an antidromic propagated nerve impulse were determined. In normal subjects an inverse relationship was found between the axonal conduction velocity and the refractory period. ${ }^{4}$ In patients with neuromuscular diseases and neuropathies, selective disturbances of either of these parameters were present. ${ }^{5}$

The aim of the present study was to evaluate the slowing of an impulse travelling in the relative refractory period of a prior impulse in single motor units in healthy man, with regard also to further studies of motor unit pathology.

Address for reprint requests: Dr J Borg, Dept of Neurology, Karolinska Hospital, Box 60500, S-104 01 Stockholm, Sweden.

Received 14 May 1982 and in revised form 30 October 1982 Accepted 20 November 1982

\section{Materials and Methods}

The study included seven medical students aged 24-36 years, three elderly subjects 74-78 years and JB aged 33 years. All were clinically healthy and had normal maximum conduction velocity in the peroneal nerve. Electromyographic recordings were made from the short toe extensor muscle by conventional bipolar needle electrodes (DISA 9013 K0802 Electronic, Skovlunde, Denmark). Selective recordings permitting safe identification of single motor unit potentials even at maximum voluntary contraction and after supramaximum nerve stimulation were obtained in the following manner: The medical students selected for the study all had an accessory deep peroneal nerve innervating only a few short toe extensor motor units. Blocking of the main peroneal nerve enabled selective recordings from motor units of the accessory nerve to be made. For elderly subjects and JB, selective recordings were made from reinnervated motor units. Reinnervation normally occurs in the short toe extensor muscle at high age. In JB reinnervation was the result of repeated electromyographic examinations with a needle electrode over a long period of time. The motor unit potentials were amplified and displayed on a Medelec oscilloscope no 4329 and recorded on Kodak Linagraph direct print paper. The common peroneal nerve was stimulated proximally at the fibular head, and distally at the ankle, posterior or anterior to the lateral malleolus. Stimuli were delivered through surface electrodes $0.6 \mathrm{~cm}$ in diameter. The cathode was placed over the nerve and the anode $2-3 \mathrm{~cm}$ laterally or medially to the cathode. Rectangular pulse waves of $0.2 \mathrm{~ms}$ duration were used. Stimulus strength could be gradually 
changed from 0 to $100 \mathrm{~mA}$. After adjusting the electrode so that the test motor unit potential could be safely identified in sustained maximum voluntary contraction, the effort was reduced until the motor unit no longer discharged and electrical stimuli were delivered in each of the following ways:

(1) A single nerve stimulus was delivered proximally and then distally, the latency difference was calculated and the axonal conduction velocity determined.

(2) Paired electrical stimuli were delivered to the nerve at the proximal stimulus point. Stimulus strength was initially $10 \%$ above the axonal threshold at rest for the first and second stimuli, that is, the conditioning and testing stimuli. Inter stimulus time was initially $10 \mathrm{~ms}$, then $5 \mathrm{~ms}$ and then reduced by $0.2 \mathrm{~ms}$ intervals until the second evoked motor unit potential disappeared, that is, blocking took place. The strength of the testing stimulus was then increased to 25,50 and $100 \%$ above the axonal threshold unless intolerable pain was experienced. The interval between the evoked motor unit responsed was measured at each stimulus interval.

(3) Paired electrical stimuli (S1 and S2) were delivered in

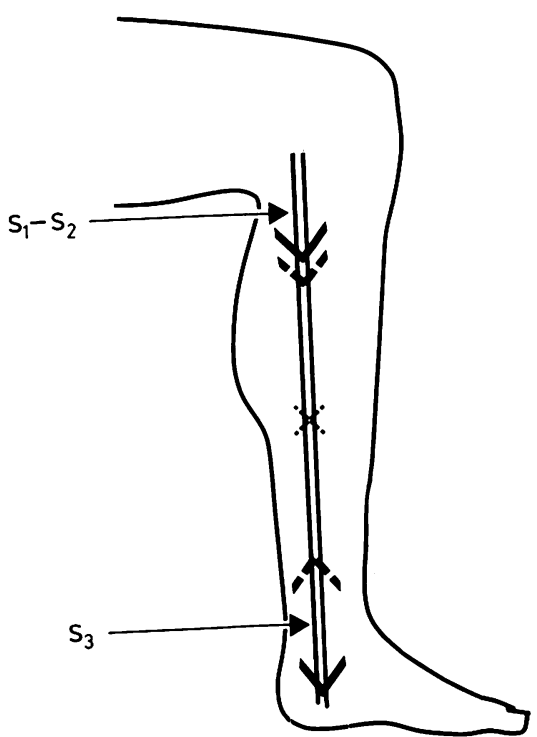

Fig 1 Paired proximal stimuli were delivered in combination with one single distal stimulus. Solid arrows represent nerve impulses evoking electromyographically recorded motor unit responses. Hatched arrows represent nerve impulses extinguished by collision. The axonal refractory period was determined at the proximal stimulus point by measuring the shortest $S 1-S 2$ interval without blocking of the motor unit response evoked by $S 2$. The delay of the $S 2$ impulse while propagated in the proximal axon segment during the relative refractory period of the $S 1$ impulse was determined by comparing the latency of the motor unit response evoked by $S 2$ with the latency of the motor unit response evoked by one single stimulus delivered at the same point as $S 2$. the same way as above. These were combined with a single distal nerve stimulus (S3) eliciting an antidromic propagated nerve impulse as illustrated in fig 1. S3 was delivered simultaneously with or at different time intervals before or after S1 so that collision between the distally evoked antidromic impulse and the first proximally evoked impulse occured at different points along the axon. This is a modification of the stimulus technique previously described by Kimura for measuring the axonal refractory period of motor nerve trunks. ${ }^{6}$ The least stimulus interval at the proximal stimulus point, without blocking of the motor unit potential evoked by the testing stimulus, S2, was measured and the axonal refractory period at the proximal test point determined. Further, the latency of the motor unit potential evoked by $\mathrm{S} 2$ was compared to the latency after a single proximal nerve stimulus. In this way, the delay of the second nerve impulse, while travelling different distances after the first impulse, could be calculated. Room temperature was $23-25^{\circ} \mathrm{C}$. Skin temperature of the lower leg and the foot was held at $32^{\circ} \mathrm{C}$ by a Disaheater (DISA, Electronic, Skovlunde, Denmark).

\section{Results}

\section{SHORTEST MOTOR UNIT RESPONSE INTERVAL}

\section{AFTER PROXIMAL NERVE STIMULATION}

When paired electrical stimuli were delivered to the peroneal nerve at the proximal stimulus point and the interstimulus time was longer than $5 \mathrm{~ms}$, the time interval between the evoked motor unit potentials was the same as between the stimuli. However, when the stimulus interval was successively reduced below $5 \mathrm{~ms}$ there was for each motor unit a certain stimulus interval below which the second motor unit: response was delayed in relation to the second stimulus. When the stimulus interval was further reduced the delay of the second evoked potential increased so that the time interval between the responses finally became fixed. This fixed interval was less than $0.5 \mathrm{~ms}$ shorter than the stimulus interval at which the delay of the second evoked response occurred. When the stimulus interval was sufficiently short, the delayed second response disappeared in an all-or-none manner. When the strength of the testing stimulus was increased, the motor unit potential reappeared in the same manner, which means that the disappearance was due to the blocking in the refractory period at the stimulus point.

Figure 2 shows the recordings of a single motor unit potential after paired proximal nerve stimulation. Stimulus strength was initially $10 \%$ above the axonal threshold at rest. In fig $2 \mathrm{a}$ the stimulus interval was $5 \mathrm{~ms}$ and the motor unit response interval was the same. In fig $2 \mathrm{~b}$ the stimulus interval was 3 $\mathrm{ms}$ and the response interval was $3.9 \mathrm{~ms}$. When the stimulus interval was further reduced, the response interval remained stable at this level. In fig $2 c$ the 


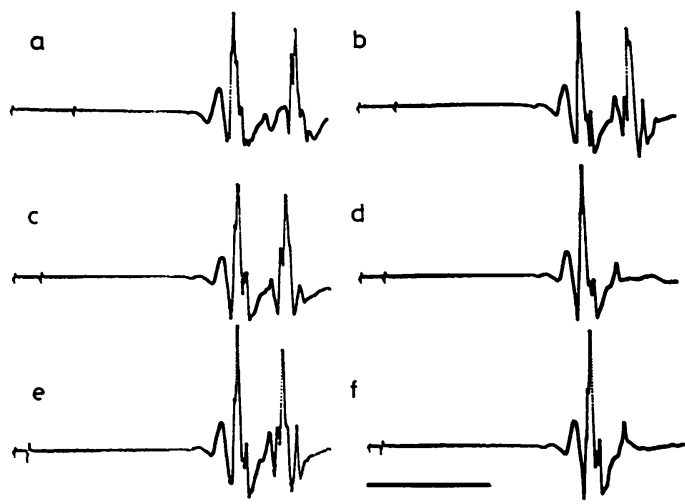

Fig 2 Motor unit response interval after proximal nerve stimulation by paired electrical stimuli. Stimulus interval in a $5 \mathrm{~ms}$, b $3 \mathrm{~ms}$, c $2.4 \mathrm{~ms}, \mathrm{~d} 2.2 \mathrm{~ms}, \mathrm{e} 1.2 \mathrm{~ms}$ and in $1.0 \mathrm{~ms}$. Response interval in a $5 \mathrm{~ms}, b, c$ and e $3.9 \mathrm{~ms}$. Second, testing, stimulus strength in a-d $10 \%$ above the axonal threshold at rest, in e-f $100 \%$ above threshold. Time bar $10 \mathrm{~ms}$.

stimulus interval was $2.4 \mathrm{~ms}$ and the response interval was $3.9 \mathrm{~ms}$. In fig $2 \mathrm{~d}$ the stimulus interval was $2 \cdot 2$ ms. The second motor unit response was not evoked. This was due to blocking in the axonal refractory period at the stimulus point because when testing stimulus strength was increased, the second response was evoked at this and even shorter stimulus intervals. This is shown in fig $2 \mathrm{e}$ where testing stimulus strength was $100 \%$ above the axonal threshold, the stimulus interval $1.2 \mathrm{~ms}$ and the response interval $3.9 \mathrm{~ms}$. At further reduction of the stimulus interval to $1.0 \mathrm{~ms}$, blocking reoccured as shown in fig $2 \mathrm{f}$.

Figure 2 shows that the second evoked and delayed motor unit potential exhibited some change. When the stimulus interval was 3-5 ms, there was often some increase in its duration, usually less than $10 \%$, and some decrease in its amplitude, usually less than $20 \%$, probably due to reduced muscle fibre conduction velocity. However, when the stimulus interval was successively decreased to less than $3 \mathrm{~ms}$, most motor units did not exhibit further changes, indicating an unchanged conduction time for the second unit potential travelling in the muscle fibres (cf Discussion).

The shortest motor unit response intervals after proximal nerve stimulation, and axonal conduction velocities were determined in 27 motor units in the seven medical students, 10 motor units in the three elderly subjects and three motor units in JB. In each subject 1-8 motor units were studied. Figure 3a shows the relationship between the shortest response intervals and the axonal conduction velocities for all 40 motor units. The shortest motor

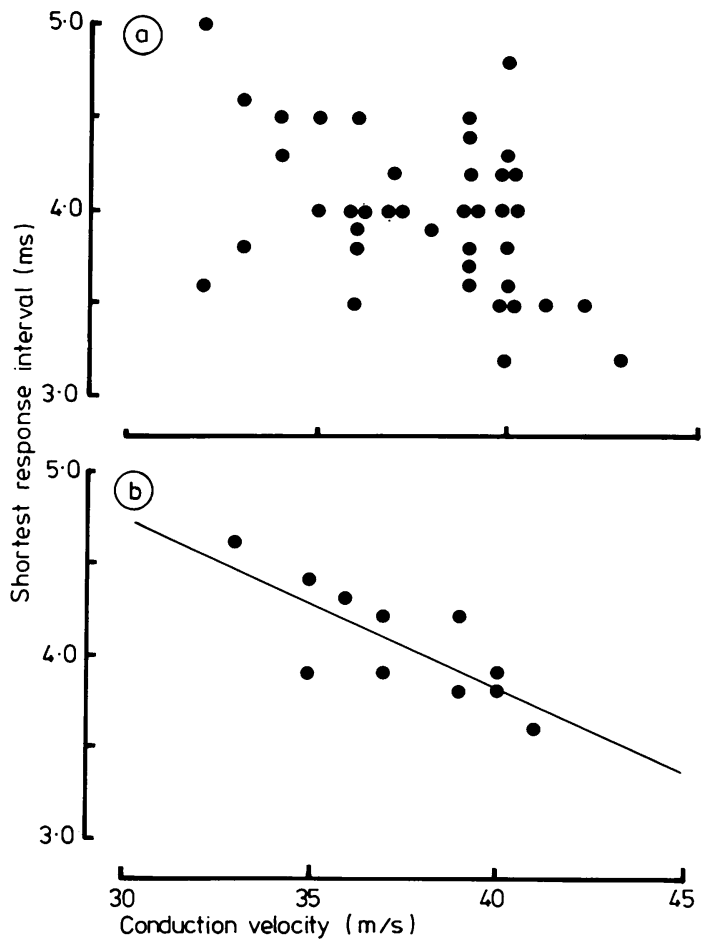

Fig 3 (a) Shortest motor unit response intervals and axonal conduction velocities of 40 motor units in 11 subjects. (b) Individual mean values of shortest motor unit response intervals and axonal conduction velocities for each of the eleven subjects. Correlation coefficient -0.78 . Regression line marked as illustration.

unit response interval ranged from 3.2 to $5 \mathrm{~ms}(\mathrm{M}=$ $3.99 \pm 0.42)$ and the axonal conduction velocity from 32 to $43 \mathrm{~m} / \mathrm{s}(M=37 \cdot 8 \pm 2 \cdot 8)$. Figure $3 \mathrm{~b}$ shows the individual mean values of the shortest motor unit response intervals and the axonal conduction velocities for each subject. The mean values of these pooled data were $4.05 \pm 0.30 \mathrm{~ms}$ and 37.5 $\pm 2.5 \mathrm{~m} / \mathrm{s}$ respectively. These data were inversely correlated, the correlation coefficient being -0.78 . The correlation was statistically significant at the $0.1 \%$ level $(p<0.01)$. No difference was found between age groups.

\section{SHORTEST MOTOR UNIT RESPONSE INTERVAL} AFTER DISTAL NERVE STIMULATION

In ten motor units the shortest motor unit response interval was determined after delivering paired electrical stimuli to the accessory or main peroneal nerve at the ankle. It was found that the shortest response interval after distal nerve stimulation was the same as after proximal nerve stimulation. 
SLOWING OF CONDUCTION AND REFRACTORY PERIOD IN THE PROXIMAL AXON SEGMENT

The slowing of the conduction of the second of paired impulses, travelling in the relative refractory period of a prior impulse in the proximal axon segment, was determined in the following manner. The latency of the motor unit potential after a single nerve stimulus was compared with the latency of the motor unit potential evoked by the second of paired stimuli delivered at the same stimulus point, when these were combined with a distal nerve stimulus a the ankle (cf Methods). The delay of the second nerve impulse occurring in the proximal half of the distance between the stimulus points could be calculated. When the interval between the paired stimuli was as long as or longer than the shortest motor unit response interval (cf above) there was no detectable delay, but when the stimulus interval was shorter there was always a delay. For 13 motor units, with stimulus intervals $1 \cdot 1-2 \mathrm{~ms}$ the delay was calculated to 0.5 to $1.2 \mathrm{~ms}(\mathrm{M}=0.87 \pm 0.22 \mathrm{~ms})$. The interval between the proximally evoked nerve impulses at the collision point about half way between the stimulus points was calculated by summing the stimulus interval and the calculated delay for each motor unit. The impulse interval ranged from 1.9 to $3.2 \mathrm{~ms}(\mathrm{M}=2.45 \pm 0.39)$ (cf below).

In the three motor units in JB the delay of the second impulse after different conduction distances was studied by altering the time relation between the proximal and distal stimuli (cf Methods). It was found that when the stimulus interval was $1.2 \mathrm{~ms}$, the delay during the first $1 \mathrm{~ms}$ of conduction was about $0.5 \mathrm{~ms}$, then slowly increased to about $1 \mathrm{~ms}$ after $5 \mathrm{~ms}$ of conduction. For longer conduction distances a slight further delay probably took place, but this was difficult to estimate due to interference with the conduction of the distal evoked ortodromic propagated potential.

For 13 motor units the axonal refractory period at the proximal stimulus point was determined by delivering paired electrical stimuli proximally combined with a distal stimulus eliciting a blocking impulse (cf Methods). Due to the delay of the second evoked motor unit response after paired stimuli (cf above), it was also possible in all these motor units to measure the refractory period without using a blocking nerve impulse. The results with and without a blocking nerve impulse were the same. The axonal refractory period when test stimulus strength was $10 \%$ above the axonal threshold at rest ranged from 1.8 to $3.3 \mathrm{~ms}(\mathrm{M}=2 \cdot 28 \pm 0.35)$.

The axonal refractory period measured at this low test stimulus strength was compared with the calculated interval between the nerve impulses after travelling half the distance between the stimulus points, as described above. In $8 / 13$ motor units the axonal refractory period was slightly shorter. In $2 / 13$ the axonal refractory period was the same and in $3 / 13$ it was slightly longer than the calculated interval. The mean values of the relative refractory period and the calculated interval were not significantly different. These results accord well with previous results in animals indicating that the velocity of the second impulse is reduced as long as it travels within the relative refractory period of the prior impulse (cf Discussion).

\section{Discussion}

Slowing of a nerve impulse travelling in the relative refractory period of a prior impulse has been described in animal peripheral nerve fibres and is of importance for their maximum discharge frequency. ${ }^{17}$ The phenomenon has also been demonstrated in central axons where functional implications were suggested. ${ }^{8}$

The present results indicate that in human alpha motor axons the slowing of a nerve impulse propagated during the relative refractory period of a prior impulse over a long distance is normally so pronounced that it is limiting for the minimum motor unit discharge interval after delivering paired elec trical stimuli to the nerve. This has not been described before.

The shortest motor unit response interval afte delivering paired electrical stimuli was 3-5 ms evere at stimulus intervals of 1-2 ms. At these short stimulus intervals there was a significant delay of the second nerve impulse travelling in the proximal axon segment. This delay was most pronounced close to the stimulus point but continued to a less extent during propagation for several milliseconds. The resulting impulse interval in the proximal axon segment corresponded well with the axonal refractory period when measured with low stimulus strength at the proximal test point. This accords well with previous findings in animal ${ }^{9}$ and human ${ }^{2}$ nerve trunks which state that recovery of the conduction velocity indicates the end of the relative refractory period as accurately as recovery of excitability.

It was not possible to determine how much further delay occurred distally in the terminal nerve twigs, the neuromuscular junction and the muscle fibres respectively. The fact that the delayed motor unit potential exhibited no changes when the stimulus interval was decreased is compatible with a further delay in the slowly conducting terminal axon branches, ${ }^{10}$ since normally there is only a slight difference in the conduction time in the terminal branches to the muscle fibres of each motor unit, ${ }^{11}$ while the delay in the muscle fibres, ${ }^{12}$ should be of 
minor importance. This is also in accord with previous findings that the refractory period of the terminal motor axons is the same as or longer than the refractory period of corresponding muscle fibres. ${ }^{13}$

The interdischarge interval was minimised to about $3 \mathrm{~ms}$ in motor units with high axonal conduction velocity and to about $5 \mathrm{~ms}$ in motor units with low axonal conduction velocity. This difference is in accord with the previously obtained difference in refractory periods of proximal axon segments. ${ }^{4}$

In voluntary contraction the minimum interval between electromyographic discharges is about $5 \mathrm{~ms}$ for motor units with high axonal conduction velocity and somewhat longer for motor units with low axonal conduction velocity. ${ }^{14}$ Further studies are in progress to determine whether axonal conduction may act as a constraint on voluntary discharge intervals (Borg, Grimby in preparation).

One consequence of the delay of the second nerve impulse is that the axonal refractory period can be measured in electromyographic recordings using paired electrical nerve stimuli without an antidromic, blocking nerve impulse, that is, in a way similar to that used in single axon studies in animals (Borg in preparation).

In conclusion, the slowing of the conduction of a nerve impulse travelling in the relative refractory period of a prior impulse over a long distance (1) is the limiting factor for the maximum motor unit discharge frequency after delivering paired electrical stimuli to the nerve, (2) offers a new parameter in studies of motor unit pathology, and (3) makes it possible to measure the axonal refractory period after nerve excitation by an electrical stimulus without using blocking nerve impulses.

\section{References}

'Tasaki I. Nervous transmission Springfield: Thomas 1953.
${ }^{2}$ Gilliat RW, Willison RG. The refractory and supernormal periods of the human median nerve. $J$ Neurol Neurosurg Psychiatry 1983;26:136-47.

${ }^{3}$ Lowitzsch K, Hopf HC. Refraktärperioden und frequency Impulsfortleitung im gemischten N. ulnaris des Menschen bei Polyneuropathien. $Z$ Neurol 1973;205: $123-44$

${ }^{4}$ Borg J. Axonal refractory period of single short toe extensor motor units in man. J Neurol Neurosurg Psychiatry 1980;43:917-24.

5 Borg J. Axonal refractory period of single short toe extensor motor units in neuropathies and neuromuscular diseases. J Neurol Neurosurg Psychiatry $1981 ; 44: 1136-40$.

${ }^{6}$ Kimura JA. Method for estimating the refractory period of motor fibres in human peripheral nerve. $J$ Neurol Sci 1976;28:485-90.

7 Paintal AS. Conduction in mammalian nerve fibres. In Desmedt, ed. New Developments in Electromyography and Clinical Neurophysiology, vol. 2, Basel: Karger, 1973:19-41.

${ }^{8}$ Kocsis JD, Swadlow HA, Waxman SG, Brill MH. Variation in conduction velocity during the relative and supernormal periods: a mechanism for impulse entrainment in central axons. Exp Neurol 1979;65:230-6.

9 Gasser HS, Erlanger J. The nature of conduction of an impulse in the relative refractory period. Am J Physiol 1925;73:613-635.

${ }^{10}$ Katz B, Miledi R. Propagation of electric activity in motor nerve terminals. Proc R Soc B 1965;161:45382.

"Buchtal F, Guld Ch, Rosenfalck P. Innervation zone and propagation velocity in human muscle. Acta Physiol Scand 1955;35:174-90.

${ }^{12}$ Stålberg E. Propagation velocity in human muscle fibres in situ. Acta Physiol Scand 1966; 70 suppl. 287:1112.

${ }^{13}$ Kopec J, Delbeke J, McComas J. Refractory period studies in a human neuromuscular preparation. $J$ Neurol Neurosurg Psychiatry 1978;41:54-64.

${ }^{14}$ Borg J, Grimby L, Hannerz J. The fatigue of voluntary contraction and peripheral electrical propagation of single short toe extensor motor units in man. In press: J Physiol (Lond). 